OPEN ACCESS

Edited by:

Udo Rudolf Markert,

University Hospital Jena, Germany

Reviewed by:

Alessandra Fiore,

Max Planck Institute of Biochemistry,

Germany

Vladimir Stanislavovich Rogovskii, Pirogov Russian National Research

Medical University, Russia

*Correspondence:

Chaogang Yang

ycg@whu.edu.cn

Jing Yang

13507182023@163.com

tThese authors have contributed equally to this work

Specialty section:

This article was submitted to Immunological Tolerance and Regulation,

a section of the journal

Frontiers in Immunology

Received: 13 August 2021 Accepted: 06 October 2021

Published: 21 October 2021

Citation:

Ding J, Zhang Y, Cai X, Diao L, Yang C and Yang J (2021) Crosstalk Between

Trophoblast and Macrophage at the Maternal-Fetal Interface: Current Status and Future Perspectives.

Front. Immunol. 12:758281. doi: 10.3389/fimmu.2021.758281

\section{Crosstalk Between Trophoblast and Macrophage at the Maternal-Fetal Interface: Current Status and Future Perspectives}

\author{
Jinli Ding ${ }^{1 \dagger}$, Yan Zhang $^{2 \dagger}$, Xiaopeng $\mathrm{Cai}^{3 \dagger}$, Lianghui Diao ${ }^{4}$, Chaogang Yang $^{3 *}$ \\ and Jing Yang ${ }^{1 *}$ \\ ${ }_{1}^{1}$ Reproductive Medical Center, Renmin Hospital of Wuhan University, Hubei Clinic Research Center for Assisted \\ Reproductive Technology and Embryonic Development, Wuhan, China, ${ }^{2}$ Department of Clinical Laboratory, Renmin Hospital \\ of Wuhan University, Wuhan, China, ${ }^{3}$ Department of Gastrointestinal Surgery, The Clinical Medical Research Center of \\ Peritoneal Cancer of Wuhan, Hubei Key Laboratory of Tumor Biological Behaviors, Hubei Cancer Clinical Study Center, \\ Zhongnan Hospital of Wuhan University, Wuhan, China, 4 Shenzhen Key Laboratory of Reproductive Immunology for \\ Periimplantation, Shenzhen Zhongshan Institute for Reproduction and Genetics, Shenzhen Zhongshan Urology Hospital, \\ Shenzhen, China
}

The immune tolerance microenvironment is crucial for the establishment and maintenance of pregnancy at the maternal-fetal interface. The maternal-fetal interface is a complex system containing various cells, including lymphocytes, decidual stromal cells, and trophoblasts. Macrophages are the second-largest leukocytes at the maternal-fetal interface, which has been demonstrated to play essential roles in remodeling spiral arteries, maintaining maternal-fetal immune tolerance, and regulating trophoblast's biological behaviors. Many researchers, including us, have conducted a series of studies on the crosstalk between macrophages and trophoblasts at the maternal-fetal interface: on the one hand, macrophages can affect the invasion and migration of trophoblasts; on the other hand, trophoblasts can regulate macrophage polarization and influence the state of the maternal-fetal immune microenvironment. In this review, we systemically introduce the functions of macrophages and trophoblasts and the cell-cell interaction between them for the establishment and maintenance of pregnancy. Advances in this area will further accelerate the basic research and clinical translation of reproductive medicine.

Keywords: maternal-fetal interface, trophoblast, macrophage, pregnancy, immune tolerance

\section{INTRODUCTION}

The establishment and maintenance of normal pregnancy is a complex process involving multiple cells and various molecules. Among them, the precise regulation at the maternal-fetal interface plays a pivotal role. The maternal-fetal interface comprises decidual immune cells, stromal cells, and trophoblasts, characterized by maintaining the defense against possible pathogens and immune tolerance to the allogeneic fetus (1). Increasing evidence demonstrates that interaction among these cellular components can influence the maternal-fetal interactive dialogue, thereby participating in 
the regulation of the pregnancy program (1). Therefore, exploring the cell-cell crosstalk at the maternal-fetal interface will help us further understand pregnancy's physiological and pathological processes.

During early pregnancy, $30-40 \%$ of the decidual cells are leukocytes, including macrophages, natural killer (NK) cells, B cells, T cells, and dendritic cells (2). Macrophages, as antigenpresenting cells, comprise $20-30 \%$ of the leukocytes at the maternal-fetal interface (3), which participate in the complex regulation at the maternal-fetal interface by regulating the secrete cytokines, phagocytosis, and immune balance (4). Besides their effects on vascular remodeling, macrophages are actively associated with trophoblasts invasion and pregnancy maintenance (5). Macrophages could be divided into classically activated (M1) and alternatively activated (M2) subtypes based on their cytokine production and function (5). The polarization states of decidual macrophages undergo dynamic changes to changing microenvironment at different gestational ages, adjusting to the different stages of fetal development. It has been demonstrated that aberrantly activated macrophages at the maternal-fetal interface may be closely related to various pregnancy complications, including miscarriage, preeclampsia, preterm birth, fetal growth restriction, or demise (6-9). Accumulating evidence indicates that the polarization of macrophages is regulated by a variety of cytokines, chemokines, sex hormones, and cell-cell interactions at the maternal-fetal interface, among which trophoblasts exert pivotal roles $(4,10,11)$. Trophoblasts are also important elements at the maternal-fetal interface, which invade the maternal myometrium and direct contact with maternal decidual stromal cells (12). Sufficient trophoblasts invasion could facilitate maternal spiral artery remodeling and placental blood flow, providing a favorable embryo implantation environment (12). Previous studies showed that the maternal microenvironment temporally and spatially controls trophoblast invasion at the maternal-fetal interface, including arteries, glands, decidual NK cells, macrophages and stromal cells (13). Trophoblasts can respond to various mediators secreted by polarized macrophages to regulate their biological behaviors (6, 14-16), while soluble cytokines produced by trophoblasts could induce macrophages polarization (17-19), involving in the regulation of normal pregnancy. Previously, our group conducted a series of studies on the crosstalk between macrophages and trophoblasts at the maternal-fetal interface: on the one hand, trophoblasts can regulate the polarization of the M2 macrophages by secreting IL-6 (20); on the other hand, M2 macrophages can promote the invasion and migration of trophoblasts by secreting G-CSF, thereby regulating the establishment and maintenance of normal pregnancy (21). In addition, macrophages could also secrete exosomes and deliver miRNAs to target and regulate the invasion and migration capabilities of trophoblasts, thereby participating in the occurrence of recurrent spontaneous abortion (RSA) (22). Although, the understanding of the interaction between trophoblasts and macrophages at the maternal-fetal interface is still insufficient and need to be further explored.
In the present review, we aimed to summarize the crosstalk between macrophages and trophoblasts at the maternal-fetal interface and the functions in normal pregnancy and pregnancy complications, providing the latest progress in the field and bringing more profound ideas to researches.

\section{THE ROLE OF MACROPHAGES AT THE MATERNAL-INTERFACE}

Generally, macrophages are classified as M1 and M2 phenotypes based on the different function and cytokine production, but in fact, there is a full spectrum of macrophage between M1 and M2 (2). Therefore, macrophages are highly plastic and heterogeneous, here we just focus on M1 and M2 as typical subtypes to explore their functions. M1 could be induced by tolllike receptor ligands, bacterial lipopolysaccharide (LPS) or other cytokines (including TNF- $\alpha$, IFN- $\gamma$, and GM-CSF), which produces reactive oxygen species and high level of IL-1 $\beta$, IL- 6 , IL-12 and IL-23 $(3,4)$. M2 can be polarized by cytokines like IL4, IL-13, IL-10, immune complexes, LPS and M-CSF, producing high levels of TGF- $\beta$ and IL-10. M2 plays important roles in clearing apoptotic cells, tissue repair and remodeling (4-6). M2 can be further divided into four subtypes, including M2a, M2b, $\mathrm{M} 2 \mathrm{c}$ and M2d. M2a-M $\varphi$ could be induced by IL-4 and IL-13, which expresses high level of CD206, TGF- $\beta$, insulin-like growth factor (IGF) and IL-1 receptor (IL-R) (7). M2b-M $\varphi$ can be induced by immune complexes plus LPS or IL-1 $\beta$, which expresses and secretes proinflammatory cytokine including TNF- $\alpha$, IL- 6 and IL- $1 \beta$, and anti-inflammatory cytokine IL-10 (8). By contrast, $\mathrm{M} 2 \mathrm{c}-\mathrm{M} \varphi$ is induced by IL-10 and releases high levels of IL-10 and TGF- $\beta$, exerting the function of phagocytosis of apoptotic cells (4). In addition, $\mathrm{M} 2 \mathrm{~d}-\mathrm{M} \varphi$, also known as tumor-associated macrophages, can be induced by IL-6, or A2 adenosine receptor plus toll-like receptor ligands, mainly characterized by high expression level of TGF- $\beta$, IL-10 and vascular endothelial growth factor (VEGF), playing essential roles in cancer metastasis and angiogenesis (9).

In the context of reproduction, macrophages are present in the endometrium, decidua and placenta, which comprise $20-30 \%$ of the leukocytes. Affected by estrogen and progesterone, the number of macrophages fluctuates during the menstrual cycle $(10,11)$. During the embryo implantation window, macrophages exhibit M1 phenotype. As trophoblasts implant and invade the endometrium, macrophages transform into a mixed M1/M2 type, which lasts to the first trimester and the early stage of the second trimester (12). At the second trimester, macrophages are polarized to the M2 phenotype to prevent rejection of the fetus by the maternal system and maintain fetal growth until delivery (13). At the time of delivery, the macrophages appear to be M1 subtype (14). Although increasing evidence has demonstrated the roles and distribution of decidual macrophages (DMs), the classification and subtypes of DMs are still controversial. Based on the expression level of CD11c and CD209, DMs are divided into CD $11 c^{\text {low }}$ and CD $11 c^{\text {high }}$ subgroups (15), or CD209 ${ }^{-}$and CD209 $9^{\text {high }}$ macrophages (16). Jiang et al. divided DMs into three 
subtypes depending on the expression of CCR2 and CD11c, including $\mathrm{CCR} 2^{-} \mathrm{CD} 11 \mathrm{c}^{\mathrm{low}}, \mathrm{CCR}^{-} \mathrm{CD} 11 \mathrm{c}^{\text {high }}$ and $\mathrm{CCR} 2^{+} \mathrm{CD} 11 \mathrm{c}^{\text {high }}(17)$. Evidence has demonstrated that $\mathrm{M} 2-\mathrm{M} \varphi$ dominates at the maternal-fetal interface, maintaining the immune-suppressive environment toward the fetus (18).

Increasing studies demonstrate that macrophages play essential roles in the establishment and maintenance of normal pregnancy, including spiral artery remodel, apoptotic cell phagocytosis and trophoblasts functions (19-21). The polarization balance between $\mathrm{M} 1-\mathrm{M} \varphi$ and $\mathrm{M} 2-\mathrm{M} \varphi$ is important for various processes of normal pregnancy. Conversely, the dysregulated macrophages polarization is associated with a variety of pregnancy complications, including RSA, preeclampsia (PE), fetal growth restriction and preterm labor $(14,22-25)$. The increased number of $\mathrm{M} 1-\mathrm{M} \varphi$ and the decreased of $\mathrm{M} 2-\mathrm{M} \varphi$ at the maternal-interface is related with RSA (23) and PE (19), accompanied by a decrease in antiinflammatory cytokines and an increase in pro-inflammatory cytokines. In addition, DMs differentiated into $\mathrm{M} 1-\mathrm{M} \varphi$ is related to spontaneous preterm labor (14). Animal experiments demonstrate that induction of M1 macrophages polarization increases embryo resorption (26), while suppressing M1 macrophages polarization alleviates the opposite effect (27). Moreover, macrophages have high plasticity, and their polarization and function are affected by the surrounding environment (4). It has been reported that hormones, chemokines, cytokines, growth factors, and the crosstalk between macrophages and related cells are involved in the regulation (28). In addition, there is growing evidence confirming the microbiome colonization at the maternal-fetal interface $(29,30)$. Compounds originating from the microbiome might bind to aryl hydrocarbon receptor (AhR), which is widely expressed in adaptive and innate immune cells including macrophages (31). Indeed, AhR plays an important role in regulating macrophage responsiveness and the expression of $\mathrm{AhR}$ in macrophage is essential for homeostasis and inflammatory responses $(31,32)$.

\section{THE ROLE OF TROPHOBLASTS AT THE MATERNAL-INTERFACE}

In the human placenta, there are three trophoblast subpopulations: the cytotrophoblasts (CTBs), syncytiotrophoblasts (STBs) and extravillous cytotrophoblasts (EVTs) (33). After embryo implantation, the outermost layer of the blastocysts transforms into mononuclear CTBs. Proliferative CTBs make up the primary villi, then the primary villi further differentiates into secondary mesenchymal villi and mature tertiary villi. Cell fusion of villous CTBs generates STBs, which plays important roles in transport of oxygen and nutrients, production of pregnancy hormones and clearance of fetal waste products (34). STBs of the floating villi represent the transport units of the human placenta, while anchoring villi transforms into another trophoblast type, namely EVTs (35). EVTs are migratory and invasive trophoblasts, which can migrate into decidual lymphatics, veins and glands, participating in placental embedment and fetal development (36-38). Defects in spiral artery remodeling, and failure in the interactions of EVTs with uterine vessels might lead to pregnancy complications, as lower numbers of EVTs in lymphatic and venous vessels is found in RSA (37). Invasive EVTs remodel the maternal spiral arteries, ensuring an adequate maternal blood supply for normal fetal growth and development. The process reduces maternal blood pressure and flow velocity, meeting the increased uteroplacental perfusion required by the developing fetus (39). The arteries remain narrow at the openings, and blood enters the intervillous space at a higher velocity, which leads to damaged villi structure and impaired placental function (40). Trophoblast invasion is temporally and spatially controlled by the maternal uterus environment, including arteries, glands, decidual NK cells, macrophages and stromal cells. Insufficient EVTs invasion has been reported to be related with pregnancy complications including RSA, PE, stillbirth and fetal intrauterine growth restriction (41-44), while excessive invasion also might lead to pathological condition such as placenta accrete (45). In addition, trophoblasts serve as a member of innate immune system at the maternal-fetal interface, as trophoblasts could recognize and respond to viral and bacterial products (46), and trophoblasts might integrate microbial-derived signals via alternative pathways mediating the response to TLRs or epigenetic modifications (47, 48). Therefore, exploring the factors affecting trophoblasts functions is of great significance for further understanding of the normal pregnancy process and the pathogenesis of pregnancy complications.

\section{CROSSTALK BETWEEN TROPHOBLASTS AND MACROPHAGES AT THE MATERNAL-FETAL INTERFACE}

In this section, we summarized the interaction between trophoblasts and macrophages, and the role in the establishment and maintenance of normal pregnancy and pathological pregnancy (Figure 1).

\section{The Effect of Macrophages on Trophoblasts Functions}

Precise regulation of the proliferation, apoptosis, migration and invasion of trophoblasts are essential for the establishment and maintenance of pregnancy. In local microenvironment, abundant DMs are observed at the implantation site and the invasive front of EVTs (49). Thence, paracrine activity of macrophages exerts an important role in biological function of trophoblasts (15). Results from rhesus monkey indicated that the growth and differentiation of embryonic trophoblasts were regulated by macrophages (50). Here, we will elaborate on the effect of macrophages on proliferation, apoptosis, migration and invasion of trophoblasts in recent years.

\section{Proliferation and Apoptosis}

Trophoblast apoptosis may induce inflammatory events that might initiate trophoblasts dysfunction, leading to pregnancy 


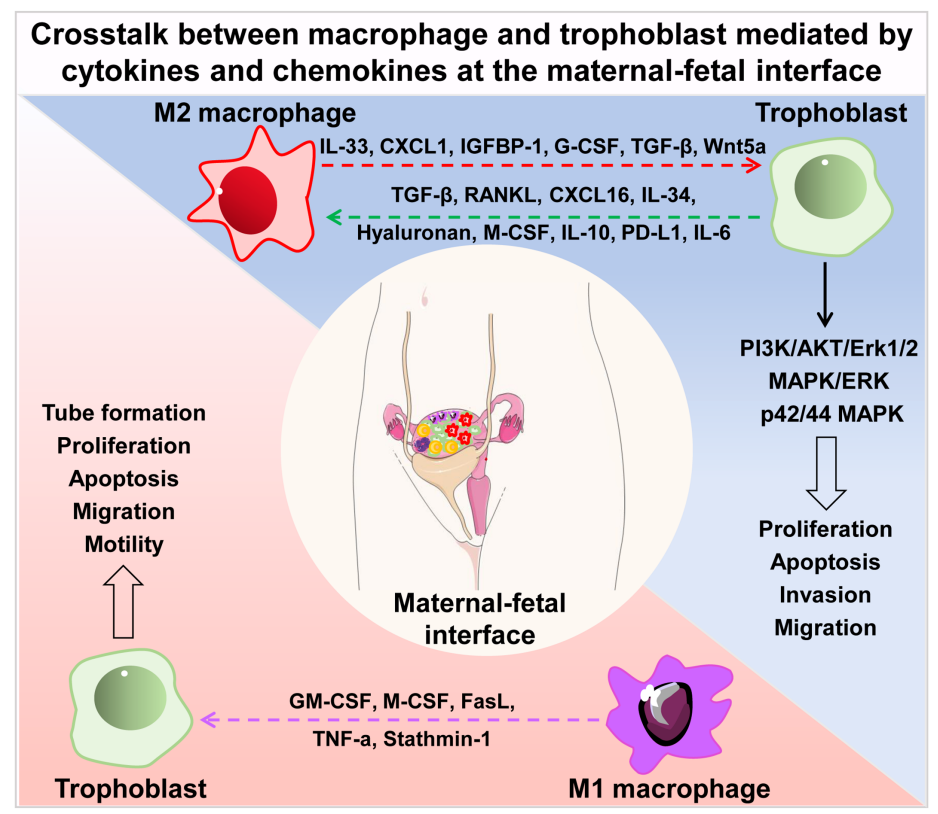

FIGURE 1 | Crosstalk between macrophage and trophoblast mediated by cytokines and chemokines at the maternal-fetal interface. Cytokines and chemokines secreted by M2 macrophages (IL-33, CXCL1, IGFBP1, G-CSF, TGF- $\beta$, Wnt5a) regulate the proliferation, apoptosis, invasion and migration of trophoblast via PI3K AKT/Erk1/2, MAPK/ERK and p42/44 MAPK signal pathways. Trophoblast- derived secretory factors (TGF- $\beta$, RANKL, CXCL16, IL-34, hyaluronan, M-CSF, IL-10, PD-L1, IL-6) drive M2 macrophage polarization in turn. Soluble molecules secreted by M1 macrophages (GM-CSF, M-CSF, FasL, TNF-a, Stathmin-1) influence the tube formation, proliferation, apoptosis, migration and motility of trophoblast. IL, interleukin; CXCL, chemokine (C-X-C motif) ligand; IGFBP1, insulin-like growth factor-binding protein-1; G-CSF, granulocyte colony stimulating factor; TGF- $\beta$, transforming growth factor $\beta$; RANKL, receptor activator of NF- $\kappa \mathrm{B}$ ligand; M-CSF, macrophage colony stimulating factor; PD-L1, programmed cell death 1 ligand 1; GM-CSF, granulocyte-macrophage colony stimulating factor; FasL, factor associated suicide ligand; TNF- $\alpha$, tumor necrosis factor $\alpha$.

complications $(23,51)$. Macrophages with different polarization statues have different effects on the motility and tube formation of trophoblasts: M1 macrophage exerted an inhibitory role, while M2 macrophages had the opposite effect (52). The Fas/FasL system is one of the major apoptotic pathways in tissues and cells. Results from our and others' group demonstrated that macrophages could induce trophoblasts apoptosis via FasL (23, 53). In addition, IL-33, as a member of the IL-1 family, has been demonstrated to promote the proliferation of cell column trophoblasts, villous cytotrophoblasts and primary trophoblasts, which was mediated by PI3K/AKT and MAPK/ ERK signaling (54). Moreover, previous report also showed that macrophages could promote trophoblasts proliferation by secreting Wnt5a via p42/44 MAPK pathway (55). Meanwhile, decidual cells stimulated with pro-inflammatory cytokines including TNF- $\alpha$ and IL- $1 \beta$ significantly strengthened the promotion effect of macrophage-induced caspase-dependent trophoblasts apoptosis via GM-CSF and M-CSF (56).

\section{Invasion and Migration}

The regulatory effects of macrophages on the invasion and migration of trophoblasts are depend on their polarization states. Renaud et al. demonstrated that nonactivated macrophages had no effect on trophoblasts invasion, while macrophages activated by LPS inhibited the ability of trophoblasts to invade through extracellular matrix in vitro (57), and the effect could be reversed by IL-10 (58). In addition, M1 macrophage-derived TNF-a reduced the expression of stathmin-1 in trophoblasts, regulating the proliferation and invasion ability, via E-cadherin/ $\beta$-catenin pathway (44). TGF- $\beta$ is a soluble mediator produced by M2 macrophages, and our recent research shows that TGF- $\beta$ secreted by M2 macrophage could induce trophoblast migration and invasion (59). Human leukocyte antigen G5 (HLA-G5) has been reported to play essential roles in immune tolerance during normal pregnancy. HLA-G5 is not expressed in the DMs, while secreted HLA-G5 could be detected in the exosomes of primary mononuclear cytotrophoblast cells and placental explants of first trimester (60). It has been confirmed that trophoblasts-derived HLA-G5 could difference monocytes into $\mathrm{M} 2-\mathrm{M} \varphi$, which in turn promotes the invasion ability of trophoblasts via secreting CXCL1 (61). Glycodelin-A is a glycoprotein abundantly present in the decidua, playing an important role in immune cell regulation at the maternal-fetal interface (62). Glycodelin-A could induce the differentiation of monocytes towards DMs-like phenotype by binding to Siglec-7, which promotes trophoblast invasion via the production of insulin-like growth factor-binding protein 1 (IGFBP-1) (63), a regulator of trophoblast functions and angiogenesis during pregnancy (64). Medroxyprogesterone is widely used for the 
treatment of endometrium cancer, abnormal uterine bleeding and secondary amenorrhea (65). Recently, it has been proved that medroxyprogesterone could drive monocyte differentiation toward M2 macrophages via ERK phosphorylation, which promoted the invasion activity of trophoblasts and the decidualization of endometrial stromal cells (66). Granulocyte colony-stimulating factor (G-CSF) is secreted by multiple cells including macrophages $(67,68)$ and placental villous trophoblasts (69). Increasing evidence demonstrates the therapeutic effect of G-CSF in RSA, repeated implantation failure, luteinized unruptured follicle syndrome and women with thin endometrium (70-72). Previously, by using a cocultured model, our study showed that trophoblasts-derived IL-6 promoted M2 macrophage polarization (73), which in turn induced the epithelial-mesenchymal transition of trophoblasts, thereby promoting migration and invasion though secreting G-CSF to activate PI3K/AKT/Erk1/2 signaling pathway (68).

\section{The Effect of Trophoblasts on Macrophages Polarization}

Macrophage polarization is a complex process and plastic to the environment surrounding or in contact with them, which is involved in multiple factors including cytokines, chemokines, growth factors, etc. Among which, cell-cell interaction exerts a vital role. DMs and EVTs are accumulated at the implantation site, and factors secreted by trophoblasts play important roles in regulating the differentiation and function of macrophages. However, the factors involved in this process has not been elucidated. In this part, we mainly focus on the effect of trophoblasts on the differentiation and function of macrophages.

Trophoblast debris have been reported to modulate the expression of cytokines in macrophages, upregulating the expression of programmed death-1 ligand 1 (PD-L1), indoleamine 2,3-dioxygenase (IDO), and anti-inflammatory cytokines including IL-1Ra, IL-6, and IL-10, while reducing the expression of costimulatory molecules (B7H3, CD40, CD80 and CD86), MHC-II molecules, IL-8 receptors, inter-cellular adhesion molecule 1 (ICAM-1), monocyte chemoattractant protein-1 (MCP-1), and pro-inflammatory cytokines including IL-8, IL-1 $\beta$, and IL-12p70 (74). Aldo et al. reported that trophoblast-derived TGF- $\beta$ could induce monocyte differentiation into $\mathrm{CD} 14^{+} / \mathrm{CD} 16^{+}$macrophages, accompanied by high secretion levels of IL-1 $\beta$, IL-10, and interferon-inducible protein-10 (IP-10), and increased phagocytosis capacity (75). By secreting IL-10 and M-CSF, trophoblasts could induce M2 macrophages polarization, sharing the phenotype of $\mathrm{CD} 163^{+} \mathrm{CD} 206^{+} \mathrm{CD} 209^{+}$and producing high level of CCL18 and IL-10 (76). IL-34 is a second ligand for the M-CSF receptor, which is involved in the maintenance and development of other macrophage subsets, such as osteoclasts (77) and Langerhans cells (78). IL-34 is produced by both decidual stromal cells and trophoblasts, which could polarize monocytes into M2 macrophages with the similar cytokine secretion pattern with DMs (79). Recently, our group has demonstrated that trophoblasts-derived IL-6 could induce M2 macrophages polarization by activating STAT3 signal pathway, with high expression of CD206, CCL18, IL-10 and TGF- $\beta$ (73). In addition, CXCL16 derived from trophoblasts differenced monocytes into M2 macrophages by binding to CXCR6, which exhibited decreased IL-15 production, facilitating the inactivation of $\mathrm{NK}$ cells and restricting the cytotoxicity of NK cells (80).

Moreover, it has been demonstrated that PD-1/PD-L1 pathway exerts an important role in regulation of immune cell homeostasis, especially macrophage polarization (26) and T cells activation (81). PD-L1 was reported to be expressed in the trophoblasts of normal placenta, and decreased expression of PD-L1 was confirmed in pregnancy complications such as RSA (26). PD-L1 expression/secretion of trophoblasts could be promoted by IFN- $\beta$, inducing PD-1/PD-L1-mediated M2 macrophage polarization (82). Similar to PD-L1, trophoblastderived HLA-G5 could drive M2 macrophages polarization manifested by increased IDO 1 and IL-6 expression (61). In addition to cytokines and chemokines, the regulatory roles of other factors have been reported. Hyaluronan (HA) is an unbranched polymer and widely present in the extracellular matrix of mammalian tissues. Decreased expression of HA was detected in villi of miscarriage patients (83). Wang et al. demonstrated that HA derived from trophoblasts could induce M2 macrophages polarization by interacting with CD44 via PI3K/AKT-STAT3/STAT-6 pathway (84). It has been indicated that HA are divided into four subtypes depending on the molecular weight, high molecular weight HA, medium molecular weight $\mathrm{HA}$, low molecular weight $\mathrm{HA}$ and $\mathrm{HA}$ oligomers (85). HA with different molecular weight might have different effects on macrophage polarization, low molecular weight HA drives M1 macrophage polarization featured with increased expression of CD80, iNOS2, TNF- $\alpha$, NO and IL-12 $\beta$, while high molecular weight $\mathrm{HA}$ induces M2 macrophage polarization charactered by increased expression of Arg1, MRC1, IL-10 and enhanced arginase activity $(86,87)$. As mentioned above, AhR is expressed in macrophages and plays an important role in regulating macrophage responsiveness (31, 32). Indeed, the expression of $A h R$ in trophoblasts has been confirmed in 2018 (88). Therefore, AhR might play a role in the pregnancy program via regulating the crosstalk between trophoblasts and macrophages. What's more, lower expression of placental AhR was found in unexplained RSA women, and the activation of AhR might impair the proliferation and migration of trophoblasts (89).

Receptor activator of NF- $\mathrm{B}$ ligand (RANKL) and its tumor necrosis factor (TNF)-family receptor RANK exert important roles in lymph node formation, bone remodeling, thymic microenvironment establishment, and mammary gland development during pregnancy. RANKL derived from trophoblasts could drive macrophage polarization to M2 subtype via activating AKT/STAT6-Jmjd3/IRF4 signaling pathway, and decreased RANKL in trophoblasts and RANK on DMs was observed in patients suffered from miscarriage (90). By using knockout mice, the authors further demonstrated that $\mathrm{RANKL}^{-/-}$mice displayed macrophage dysfunction and 
increased fetal loss, while adoptive transfer of $\mathrm{RANK}^{+}$ macrophages could relieve the fetal loss induced by macrophage depletion (90).

Altogether, results from the above studies confirm the role of trophoblasts on M2 macrophage polarization, which were based on trophoblasts from healthy pregnancy women or trophoblast cell lines. However, it is not known whether these roles were maintained in a pathological condition, as trophoblast dysfunction was related to pregnancy complications such as unexplained RSA. A previous study from an ex vivo model demonstrated that trophoblasts from unexplained RSA could promote an M2-like phenotype, indicating that trophoblasts from unexplained RSA had similar functional and proteomic properties with trophoblasts from healthy women (91). Cluster of differentiation 74 (CD74) was a high-affinity binding protein for the inflammatory cytokine macrophage migration inhibitory factor, which was released by trophoblasts to regulate monocyte activity (92). Przybyl et al. demonstrated that the adhesion of macrophages lacking CD74 to trophoblasts was decreased, exhibiting the pro-inflammatory phenotype when co-cultured with trophoblasts (24).

\section{THE ROLE OF EXTRACELLULAR VESICLES IN CROSSTALK BETWEEN MACROPHAGES AND TROPHOBLASTS AT THE MATERNAL-FETAL INTERFACE}

In addition to soluble factors, extracellular vesicles (EVs) are also demonstrated to be another important medium in communication network at the maternal-fetal interface in recent years (93). EVs, including microvesicles, exosomes and apoptotic bodies, are intricately involved in cell-cell communication, which are released by various types of cells, including macrophages and trophoblasts. EVs contain abundant molecules like lipids, proteins, DNA, mRNAs, long non-coding RNAs (LncRNA) and miRNAs, which are delivered to target cells (94). EVs could be detected in multiple biofluids including milk, urine, blood, saliva and amniotic fluid (95). EVs concentration in circulation is increased in pregnant women, with 5-folds higher than non-pregnant women (96), which could be detected in maternal plasma at 6 weeks of gestational age (97). Trophoblasts-released EVs are taken up by cells of maternal vascular and immune systems, regulating the maternal physiological system to adapt to the pregnancy process (93). Placental EVs has been proven to exert an important role in maintaining maternal-fetal immune tolerance via repression of modulatory signals in the maternal immune system and suppression of natural killer cells and T-lymphocyte activation (98). The release of EVs by EVTs has been confirmed as HLA-G EVs were detected in the maternal circulation during pregnancy (99). In this section, we mainly summarized the latest progress about the role of EVs in crosstalk between macrophages and trophoblasts at the maternal-fetal interface (Figure 2).

In fact, little is known about EVs-mediated crosstalk between macrophages and trophoblasts up to now. Atay et al. firstly reported that trophoblast-derived exosomes could recruit and educate monocytes to produce G-CSF, granulocyte/monocyte colony-stimulating factor (GM-CSF), IL- $1 \beta$, IL- 6 , TNF- $\alpha$ and Serpin-E1, in a cell-contact-independent manner, which were necessary for embryo implantation, stromal remodeling and angiogenesis (100). These authors further addressed the mechanism that IL-1 $\beta$ induction by exosome-associated fibronectin (101). EVs released by macrophages in turn modulate trophoblast's function. Holder et al. reported that macrophages-derived exosomes were internalized by human placenta in a time- and dose-dependent manner, via clathrindependent endocytosis, inducing the release of proinflammatory

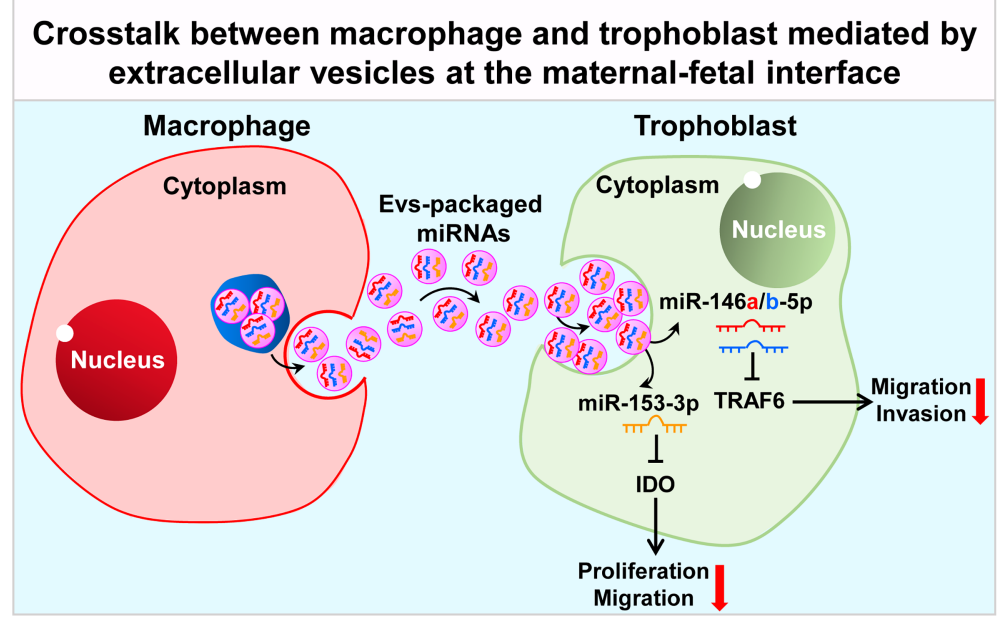

FIGURE 2 | Crosstalk between macrophage and trophoblast mediated by extracellular vesicles at the maternal-fetal interface. Macrophage-derived extracellular vesicles affect the expression of TRAF6 and IDO in trophoblast by delivering miR-146a-5p, miR-146b-5p and miR-153-3p, thereby affecting their invasion and migration capabilities, participating in the pathological process of RSA. TRAF6, TNF receptor associated factor 6; IDO, indoleamine 2, 3-dioxygenase. 
cytokines, including IL-6, IL-8 and IL-10 (102). EVs exert biological functions mainly through the biologically molecules they conduct. For example, EVs released from DMs could deliver miR-153-3p to target IDO, inhibiting trophoblasts proliferation and migration via STAT3 pathway and contributing to occurrence of unexplained RSA (103). Recently, our group reported that EVs derived from $\mathrm{M} 1-\mathrm{M} \varphi$ could deliver miR146a-5p and miR-146b-5p to target TNF receptor associated factor 6 (TRAF6), suppressing trophoblast migration and invasion and contributing to the development of RSA (104). However, more evidence is needed to confirm the role of EVs in the establishment and maintenance of pregnancy.

\section{CONCLUSIONS AND PERSPECTIVES}

Taken together, current evidence suggests that trophoblasts and macrophages can establish extensive connections at the maternal-fetal interface and thus participate in regulating the physiological and pathological processes of pregnancy. Polarized macrophages can influence the biological behavior (including proliferation, apoptosis, invasion and migration) through the secretion of various cytokines and chemokines. Reciprocally, trophoblasts can also regulate the polarization state of macrophages through a variety of mechanisms, thus affecting the establishment and maintenance of immune tolerance microenvironment at the maternal-fetal interface.

In spite of this, the interaction between trophoblasts and macrophages still needs further study: 1) As above presented, the crosstalk reported for now between trophoblasts and macrophages are mainly mediated by cytokines and chemokines. EVs, as an emerging intercellular communication medium, have been gradually proven to exert role in mediating the mutual communication between cells. However, the role and potential mechanisms of EVs in the crosstalk between macrophages and trophoblasts at the maternal-fetal interface is

\section{REFERENCES}

1. Xu L, Li Y, Sang Y, Li DJ, Du M. Crosstalk Between Trophoblasts and Decidual Immune Cells: The Cornerstone of Maternal-Fetal Immunotolerance. Front Immunol (2021) 12:642392. doi: 10.3389/ fimmu.2021.642392

2. Mosser DM, Edwards JP. Exploring the Full Spectrum of Macrophage Activation. Nat Rev Immunol (2008) 8(12):958-69. doi: 10.1038/nri2448

3. Nathan CF. Mechanisms of Macrophage Antimicrobial Activity. Trans $R$ Soc Trop Med Hyg (1983) 77(5):620-30. doi: 10.1016/0035-9203(83)90190-6

4. Shapouri-Moghaddam A, Mohammadian S, Vazini H, Taghadosi M, Esmaeili SA, Mardani F, et al. Macrophage Plasticity, Polarization, and Function in Health and Disease. J Cell Physiol (2018) 233(9):6425-40. doi: $10.1002 /$ jcp.26429

5. Gordon S. Alternative Activation of Macrophages. Nat Rev Immunol (2003) 3(1):23-35. doi: 10.1038/nri978

6. Satoh T, Takeuchi O, Vandenbon A, Yasuda K, Tanaka Y, Kumagai Y, et al. The Jmjd3-Irf4 Axis Regulates M2 Macrophage Polarization and Host Responses Against Helminth Infection. Nat Immunol (2010) 11(10):93644. doi: 10.1038/ni.1920 rarely studied, which is an emerging field that needs to be explored urgently. 2) What' more, in addition to macrophages, there are also immune cells including $\mathrm{T}$ cells, NK cells and dendritic cells at the maternal-fetal interface, which also play important roles in regulating the biological behavior of trophoblasts. Then, whether other cells may also participate in the interaction between macrophages and trophoblasts, thereby establishing three-cell or multi-cell communication, and then participating in the establishment and maintenance of pregnancy, is also worth exploring in future research. 3) Additionally, as most of the current researches are based on cell lines, transforming the interaction of macrophages and trophoblasts into clinical research is also a direction worth studying.

\section{AUTHOR CONTRIBUTIONS}

CY and JY designed the review. JD, YZ, and XC drafted the manuscript and prepared the figures. LD helped to modify the manuscript. All authors contributed to the article and approved the submitted version.

\section{FUNDING}

This work was supported by grants from the National Key Research and Development Program of China (No. 2018YFC1002804), the National Natural Science Foundation of China (No. 82101749, 81771662, 81971356 and 81801540), the Fundamental Research Funds for the Central Universities (No. $2042021 \mathrm{kf0082}$ and 2042021kf0143), Zhongnan Hospital of Wuhan University, Excellent Doctor Fund Project (No. ZNYB2020002), and Technology and Innovation Seed Found (No. CXPY2020025).

7. Wynn TA, Vannella KM. Macrophages in Tissue Repair, Regeneration, and Fibrosis. Immunity (2016) 44(3):450-62. doi: 10.1016/j.immuni.2016.02.015

8. Yue Y, Yang X, Feng K, Wang L, Hou J, Mei B, et al. M2b Macrophages Reduce Early Reperfusion Injury After Myocardial Ischemia in Mice: A Predominant Role of Inhibiting Apoptosis. Via A20 Int J Cardiol (2017) 245:228-35. doi: 10.1016/j.ijcard.2017.07.085

9. Atri C, Guerfali FZ, Laouini D. Role of Human Macrophage Polarization in Inflammation During Infectious Diseases. Int J Mol Sci (2018) 19(6):1801 doi: 10.3390/ijms19061801

10. Toniolo A, Fadini GP, Tedesco S, Cappellari R, Vegeto E, Maggi A, et al. Alternative Activation of Human Macrophages Is Rescued by Estrogen Treatment In Vitro and Impaired by Menopausal Status. J Clin Endocrinol Metab (2015) 100(1):E50-8. doi: 10.1210/jc.2014-2751

11. Tedesco S, Adorni MP, Ronda N, Cappellari R, Mioni R, Barbot M, et al Activation Profiles of Monocyte-Macrophages and HDL Function in Healthy Women in Relation to Menstrual Cycle and in Polycystic Ovary Syndrome Patients. Endocrine (2019) 66(2):360-9. doi: 10.1007/s12020-019-01911-2

12. Das A, Sinha M, Datta S, Abas M, Chaffee S, Sen CK, et al. Monocyte and Macrophage Plasticity in Tissue Repair and Regeneration. Am J Pathol (2015) 185(10):2596-606. doi: 10.1016/j.ajpath.2015.06.001 
13. Faas MM, de Vos P. Uterine NK Cells and Macrophages in Pregnancy. Placenta (2017) 56:44-52. doi: 10.1016/j.placenta.2017.03.001

14. Xu Y, Romero R, Miller D, Kadam L, Mial TN, Plazyo O, et al. An M1-Like Macrophage Polarization in Decidual Tissue During Spontaneous Preterm Labor That Is Attenuated by Rosiglitazone Treatment. J Immunol (2016) 196 (6):2476-91. doi: 10.4049/jimmunol.1502055

15. Houser BL, Tilburgs T, Hill J, Nicotra ML, Strominger JL. Two Unique Human Decidual Macrophage Populations. J Immunol (2011) 186(4):263342. doi: 10.4049/jimmunol.1003153

16. Svensson J, Jenmalm MC, Matussek A, Geffers R, Berg G, Ernerudh J. Macrophages at the Fetal-Maternal Interface Express Markers of Alternative Activation and Are Induced by M-CSF and IL-10. J Immunol (2011) 187 (7):3671-82. doi: 10.4049/jimmunol.1100130

17. Jiang X, Du MR, Li M, Wang H. Three Macrophage Subsets Are Identified in the Uterus During Early Human Pregnancy. Cell Mol Immunol (2018) 15 (12):1027-37. doi: 10.1038/s41423-018-0008-0

18. Jena MK, Nayak N, Chen K, Nayak NR. Role of Macrophages in Pregnancy and Related Complications. Arch Immunol Ther Exp (Warsz) (2019) 67 (5):295-309. doi: 10.1007/s00005-019-00552-7

19. Zhang Q, Shan F, Li Z, Gao J, Li Y, Shen L, et al. A Prospective Study on the Changes and Clinical Significance of Pre-Operative and Post-Operative Circulating Tumor Cells in Resectable Gastric Cancer. J Transl Med (2018) 16(1):171. doi: 10.1186/s12967-018-1544-1

20. Ning F, Liu H, Lash GE. The Role of Decidual Macrophages During Normal and Pathological Pregnancy. Am J Reprod Immunol (2016) 75(3):298-309. doi: 10.1111/aji.12477

21. Okabe Y, Medzhitov R. Tissue Biology Perspective on Macrophages. Nat Immunol (2016) 17(1):9-17. doi: 10.1038/ni.3320

22. Zhang YH, He M, Wang Y, Liao AH. Modulators of the Balance Between M1 and M2 Macrophages During Pregnancy. Front Immunol (2017) 8:120. doi: 10.3389/fimmu.2017.00120

23. Ding J, Yin T, Yan N, Cheng Y, Yang J. FasL on Decidual Macrophages Mediates Trophoblast Apoptosis: A Potential Cause of Recurrent Miscarriage. Int J Mol Med (2019) 43(6):2376-86. doi: 10.3892/ ijmm.2019.4146

24. Przybyl L, Haase N, Golic M, Rugor J, Solano ME, Arck PC, et al. CD74Downregulation of Placental Macrophage-Trophoblastic Interactions in Preeclampsia. Circ Res (2016) 119(1):55-68. doi: 10.1161/ CIRCRESAHA.116.308304

25. Bezemer RE, Schoots MH, Timmer A, Scherjon SA, Erwich J, van Goor H, et al. Altered Levels of Decidual Immune Cell Subsets in Fetal Growth Restriction, Stillbirth, and Placental Pathology. Front Immunol (2020) 11:1898. doi: 10.3389/fimmu.2020.01898

26. Zhang Y, Ma L, Hu X, Ji J, Mor G, Liao A. The Role of the PD-1/PD-L1 Axis in Macrophage Differentiation and Function During Pregnancy. Hum Reprod (2019) 34(1):25-36. doi: 10.1093/humrep/dey347

27. Zhu X, Liu H, Zhang Z, Wei R, Zhou X, Wang Z, et al. MiR-103 Protects From Recurrent Spontaneous Abortion via Inhibiting STAT1 Mediated M1 Macrophage Polarization. Int J Biol Sci (2020) 16(12):2248-64. doi: 10.7150/ ijbs.46144

28. Han X, Li W, Li P, Zheng Z, Lin B, Zhou B, et al. Stimulation of Alpha7 Nicotinic Acetylcholine Receptor by Nicotine Suppresses Decidual M1 Macrophage Polarization Against Inflammation in LipopolysaccharideInduced Preeclampsia-Like Mouse Model. Front Immunol (2021) 12:642071. doi: 10.3389/fimmu.2021.642071

29. Agostinis C, Mangogna A, Bossi F, Ricci G, Kishore U, Bulla R. Uterine Immunity and Microbiota: A Shifting Paradigm. Front Immunol (2019) 10:2387. doi: 10.3389/fimmu.2019.02387

30. Mor G, Kwon JY. Trophoblast-Microbiome Interaction: A New Paradigm on Immune Regulation. Am J Obstet Gynecol (2015) 213(4 Suppl):S131-7. doi: 10.1016/j.ajog.2015.06.039

31. Shinde R, McGaha TL. The Aryl Hydrocarbon Receptor: Connecting Immunity to the Microenvironment. Trends Immunol (2018) 39 (12):1005-20. doi: 10.1016/j.it.2018.10.010

32. Goudot C, Coillard A, Villani AC, Gueguen P, Cros A, Sarkizova S, et al. Aryl Hydrocarbon Receptor Controls Monocyte Differentiation Into Dendritic Cells Versus Macrophages. Immunity (2017) 47(3):582-96 e6. doi: 10.1016/ j.immuni.2017.08.016
33. Maltepe E, Fisher SJ. Placenta: The Forgotten Organ. Annu Rev Cell Dev Biol (2015) 31:523-52. doi: 10.1146/annurev-cellbio-100814-125620

34. Aplin JD. Developmental Cell Biology of Human Villous Trophoblast: Current Research Problems. Int J Dev Biol (2010) 54(2-3):323-9. doi: $10.1387 / \mathrm{ijdb} .082759 \mathrm{ja}$

35. James JL, Carter AM, Chamley LW. Human Placentation From Nidation to 5 Weeks of Gestation. Part I: What do We Know About Formative Placental Development Following Implantation? Placenta (2012) 33(5):327-34. doi: 10.1016/j.placenta.2012.01.020

36. Moser G, Weiss G, Sundl M, Gauster M, Siwetz M, Lang-Olip I, et al. Extravillous Trophoblasts Invade More Than Uterine Arteries: Evidence for the Invasion of Uterine Veins. Histochem Cell Biol (2017) 147(3):353-66. doi: 10.1007/s00418-016-1509-5

37. Windsperger K, Dekan S, Pils S, Golletz C, Kunihs V, Fiala C, et al. Extravillous Trophoblast Invasion of Venous as Well as Lymphatic Vessels Is Altered in Idiopathic, Recurrent, Spontaneous Abortions. Hum Reprod (2017) 32(6):1208-17. doi: 10.1093/humrep/dex058

38. He N, van Iperen $\mathrm{L}$, de Jong $\mathrm{D}$, Szuhai $\mathrm{K}$, Helmerhorst FM, van der Westerlaken LA, et al. Human Extravillous Trophoblasts Penetrate Decidual Veins and Lymphatics Before Remodeling Spiral Arteries During Early Pregnancy. PloS One (2017) 12(1):e0169849. doi: 10.1371/ journal.pone. 0169849

39. Burton GJ, Woods AW, Jauniaux E, Kingdom JC. Rheological and Physiological Consequences of Conversion of the Maternal Spiral Arteries for Uteroplacental Blood Flow During Human Pregnancy. Placenta (2009) 30(6):473-82. doi: 10.1016/j.placenta.2009.02.009

40. Kingdom JC, Audette MC, Hobson SR, Windrim RC, Morgen E. A Placenta Clinic Approach to the Diagnosis and Management of Fetal Growth Restriction. Am J Obstet Gynecol (2018) 218(2S):S803-S17. doi: 10.1016/ j.ajog.2017.11.575

41. DaSilva-Arnold S, James JL, Al-Khan A, Zamudio S, Illsley NP. Differentiation of First Trimester Cytotrophoblast to Extravillous Trophoblast Involves an Epithelial-Mesenchymal Transition. Placenta (2015) 36(12):1412-8. doi: 10.1016/j.placenta.2015.10.013

42. Ding J, Cheng Y, Zhang Y, Liao S, Yin T, Yang J. The miR-27a-3p/USP25 Axis Participates in the Pathogenesis of Recurrent Miscarriage by Inhibiting Trophoblast Migration and Invasion. J Cell Physiol (2019) 234(11):1995163. doi: $10.1002 /$ jcp. 28593

43. Rugg-Gunn PJ. Epigenetic Features of the Mouse Trophoblast. Reprod BioMed Online (2012) 25(1):21-30. doi: 10.1016/j.rbmo.2012.01.012

44. Tian FJ, Qin CM, Li XC, Wu F, Liu XR, Xu WM, et al. Decreased Stathmin-1 Expression Inhibits Trophoblast Proliferation and Invasion and Is Associated With Recurrent Miscarriage. Am J Pathol (2015) 185(10):270921. doi: 10.1016/j.ajpath.2015.06.010

45. Jauniaux E, Collins S, Burton GJ. Placenta Accreta Spectrum: Pathophysiology and Evidence-Based Anatomy for Prenatal Ultrasound Imaging. Am J Obstet Gynecol (2018) 218(1):75-87. doi: 10.1016/j.ajog.2017.05.067

46. Abrahams VM, Visintin I, Aldo PB, Guller S, Romero R, Mor G. A Role for TLRs in the Regulation of Immune Cell Migration by First Trimester Trophoblast Cells. J Immunol (2005) 175(12):8096-104. doi: 10.4049/ jimmunol.175.12.8096

47. Silasi M, Mor G. Decidual Stromal Cells as Regulators of T-Cell Access to the Maternal-Fetal Interface. Am J Reprod Immunol (2012) 68(4):279-81. doi: 10.1111/aji.12006

48. Alenghat T, Osborne LC, Saenz SA, Kobuley D, Ziegler CG, Mullican SE, et al. Histone Deacetylase 3 Coordinates Commensal-Bacteria-Dependent Intestinal Homeostasis. Nature (2013) 504(7478):153-7. doi: 10.1038/ nature 12687

49. Helige C, Ahammer H, Moser G, Hammer A, Dohr G, Huppertz B, et al. Distribution of Decidual Natural Killer Cells and Macrophages in the Neighbourhood of the Trophoblast Invasion Front: A Quantitative Evaluation. Hum Reprod (2014) 29(1):8-17. doi: 10.1093/humrep/det353

50. Rozner AE, Durning M, Kropp J, Wiepz GJ, Golos TG. Macrophages Modulate the Growth and Differentiation of Rhesus Monkey Embryonic Trophoblasts. Am J Reprod Immunol (2016) 76(5):364-75. doi: 10.1111/aji.12564

51. Teirila L, Heikkinen-Eloranta J, Kotimaa J, Meri S, Lokki AI. Regulation of the Complement System and Immunological Tolerance in Pregnancy. Semin Immunol (2019) 45:101337. doi: 10.1016/j.smim.2019.101337 
52. Buckley RJ, Whitley GS, Dumitriu IE, Cartwright JE. Macrophage Polarisation Affects Their Regulation of Trophoblast Behaviour. Placenta (2016) 47:73-80. doi: 10.1016/j.placenta.2016.09.004

53. Guirelli PM, Angeloni MB, Barbosa BF, Gomes AO, Castro AS, Franco PS, et al. Trophoblast-Macrophage Crosstalk on Human Extravillous Under Toxoplasma Gondii Infection. Placenta (2015) 36(10):1106-14. doi: 10.1016/j.placenta.2015.08.009

54. Fock V, Mairhofer M, Otti GR, Hiden U, Spittler A, Zeisler H, et al. Macrophage-Derived IL-33 Is a Critical Factor for Placental Growth. J Immunol (2013) 191(7):3734-43. doi: 10.4049/jimmunol.1300490

55. Meinhardt G, Saleh L, Otti GR, Haider S, Velicky P, Fiala C, et al. Wingless Ligand 5a Is a Critical Regulator of Placental Growth and Survival. Sci Rep (2016) 6:28127. doi: 10.1038/srep28127

56. Wu ZM, Yang H, Li M, Yeh CC, Schatz F, Lockwood CJ, et al. ProInflammatory Cytokine-Stimulated First Trimester Decidual Cells Enhance Macrophage-Induced Apoptosis of Extravillous Trophoblasts. Placenta (2012) 33(3):188-94. doi: 10.1016/j.placenta.2011.12.007

57. Renaud SJ, Postovit LM, Macdonald-Goodfellow SK, McDonald GT, Caldwell JD, Graham CH. Activated Macrophages Inhibit Human Cytotrophoblast Invasiveness. Vitro Biol Reprod (2005) 73(2):237-43. doi: 10.1095/biolreprod.104.038000

58. Renaud SJ, Macdonald-Goodfellow SK, Graham CH. Coordinated Regulation of Human Trophoblast Invasiveness by Macrophages and Interleukin 10. Biol Reprod (2007) 76(3):448-54. doi: 10.1095/ biolreprod.106.055376

59. Wang J, Ding J, Zhang S, Chen X, Yan S, Zhang Y, et al. Decreased USP2a Expression Inhibits Trophoblast Invasion and Associates With Recurrent Miscarriage. Front Immunol (2021) 12:717370. doi: 10.3389/ fimmu.2021.717370

60. Kshirsagar SK, Alam SM, Jasti S, Hodes H, Nauser T, Gilliam M, et al. Immunomodulatory Molecules Are Released From the First Trimester and Term Placenta via Exosomes. Placenta (2012) 33(12):982-90. doi: 10.1016/ j.placenta.2012.10.005

61. Lee CL, Guo Y, So KH, Vijayan M, Guo Y, Wong VH, et al. Soluble Human Leukocyte Antigen G5 Polarizes Differentiation of Macrophages Toward a Decidual Macrophage-Like Phenotype. Hum Reprod (2015) 30(10):2263-74. doi: 10.1093/humrep/dev196

62. Lee CL, Lam KK, Vijayan M, Koistinen H, Seppala M, Ng EH, et al. The Pleiotropic Effect of Glycodelin-A in Early Pregnancy. Am J Reprod Immunol (2016) 75(3):290-7. doi: 10.1111/aji.12471

63. Vijayan M, Lee CL, Wong VHH, Wang X, Bai K, Wu J, et al. Decidual Glycodelin-A Polarizes Human Monocytes Into a Decidual MacrophageLike Phenotype Through Siglec-7. J Cell Sci (2020) 133(14):jcs24440. doi: $10.1242 /$ jcs. 244400

64. Santos ED, Moindjie H, Serazin V, Arnould L, Rodriguez Y, Fathallah K, et al. Preimplantation Factor Modulates Trophoblastic Invasion Throughout the Decidualization of Human Endometrial Stromal Cells. Reprod Biol Endocrinol (2021) 19(1):96. doi: 10.1186/s12958-021-00774-5

65. Ayele H, Perner M, McKinnon LR, Birse K, Farr Zuend C, Burgener A. An Updated Review on the Effects of Depot Medroxyprogesterone Acetate on the Mucosal Biology of the Female Genital Tract. Am J Reprod Immunol (2021) 86(3):e13455. doi: 10.1111/aji.13455

66. Tsai YC, Tseng JT, Wang CY, Su MT, Huang JY, Kuo PL. Medroxyprogesterone Acetate Drives M2 Macrophage Differentiation Toward a Phenotype of Decidual Macrophage. Mol Cell Endocrinol (2017) 452:74-83. doi: 10.1016/j.mce.2017.05.015

67. Chang SF, Lin SS, Yang HC, Chou YY, Gao JI, Lu SC. LPS-Induced G-CSF Expression in Macrophages Is Mediated by ERK2, But Not Erk1. PloS One (2015) 10(6):e0129685. doi: 10.1371/journal.pone.0129685

68. Ding J, Yang C, Zhang Y, Wang J, Zhang S, Guo D, et al. M2 MacrophageDerived G-CSF Promotes Trophoblasts EMT, Invasion and Migration via Activating PI3K/Akt/Erk1/2 Pathway to Mediate Normal Pregnancy. J Cell Mol Med (2021) 25(4):2136-47. doi: 10.1111/jcmm.16191

69. Scarpellini F, Klinger FG, Rossi G, Sbracia M. Immunohistochemical Study on the Expression of G-CSF, G-CSFR, VEGF, VEGFR-1, Foxp3 in First Trimester Trophoblast of Recurrent Pregnancy Loss in Pregnancies Treated With G-CSF and Controls. Int J Mol Sci (2019) 21(1):285. doi: 10.3390/ ijms 21010285
70. Eapen A, Joing M, Kwon P, Tong J, Maneta E, De Santo C, et al. Recombinant Human Granulocyte- Colony Stimulating Factor in Women With Unexplained Recurrent Pregnancy Losses: A Randomized Clinical Trial. Hum Reprod (2019) 34(3):424-32. doi: 10.1093/humrep/dey393

71. Wurfel W. Treatment With Granulocyte Colony-Stimulating Factor in Patients With Repetitive Implantation Failures and/or Recurrent Spontaneous Abortions. J Reprod Immunol (2015) 108:123-35. doi: 10.1016/j.jri.2015.01.010

72. Kamath MS, Kirubakaran R, Sunkara SK. Granulocyte-Colony Stimulating Factor Administration for Subfertile Women Undergoing Assisted Reproduction. Cochrane Database Syst Rev (2020) 1:CD013226. doi: 10.1002/14651858.CD013226.pub2

73. Ding J, Yang C, Cheng Y, Wang J, Zhang S, Yan S, et al. TrophoblastDerived IL-6 Serves as an Important Factor for Normal Pregnancy by Activating Stat3-Mediated M2 Macrophages Polarization. Int Immunopharmacol (2021) 90:106788. doi: 10.1016/j.intimp.2020.106788

74. Abumaree MH, Chamley LW, Badri M, El-Muzaini MF. Trophoblast Debris Modulates the Expression of Immune Proteins in Macrophages: A Key to Maternal Tolerance of the Fetal Allograft? J Reprod Immunol (2012) 94 (2):131-41. doi: 10.1016/j.jri.2012.03.488

75. Aldo PB, Racicot K, Craviero V, Guller S, Romero R, Mor G. Trophoblast Induces Monocyte Differentiation Into CD14+/CD16+ Macrophages. Am J Reprod Immunol (2014) 72(3):270-84. doi: 10.1111/aji.12288

76. Svensson-Arvelund J, Mehta RB, Lindau R, Mirrasekhian E, RodriguezMartinez H, Berg G, et al. The Human Fetal Placenta Promotes Tolerance Against the Semiallogeneic Fetus by Inducing Regulatory T Cells and Homeostatic M2 Macrophages. J Immunol (2015) 194(4):1534-44. doi: 10.4049/jimmunol.1401536

77. Chen Z, Buki K, Vaaraniemi J, Gu G, Vaananen HK. The Critical Role of IL34 in Osteoclastogenesis. PloS One (2011) 6(4):e18689. doi: 10.1371/ journal.pone.0018689

78. Wang Y, Szretter KJ, Vermi W, Gilfillan S, Rossini C, Cella M, et al. IL-34 Is a Tissue-Restricted Ligand of CSF1R Required for the Development of Langerhans Cells and Microglia. Nat Immunol (2012) 13(8):753-60. doi: 10.1038/ni.2360

79. Lindau R, Mehta RB, Lash GE, Papapavlou G, Boij R, Berg G, et al. Interleukin-34 Is Present at the Fetal-Maternal Interface and Induces Immunoregulatory Macrophages of a Decidual Phenotype In Vitro. Hum Reprod (2018) 33(4):588-99. doi: 10.1093/humrep/dey037

80. Wang XQ, Zhou WJ, Hou XX, Fu Q, Li DJ. Trophoblast-Derived CXCL16 Induces M2 Macrophage Polarization That in Turn Inactivates NK Cells at the Maternal-Fetal Interface. Cell Mol Immunol (2018) 15(12):1038-46. doi: 10.1038/s41423-018-0019-x

81. Salvany-Celades M, van der Zwan A, Benner M, Setrajcic-Dragos V, Bougleux Gomes HA, Iyer V, et al. Three Types of Functional Regulatory $\mathrm{T}$ Cells Control T Cell Responses at the Human Maternal-Fetal Interface. Cell Rep (2019) 27(9):2537-47 e5. doi: 10.1016/j.celrep.2019.04.109

82. Zhang YH, Aldo P, You Y, Ding J, Kaislasuo J, Petersen JF, et al. Trophoblast-Secreted Soluble-PD-L1 Modulates Macrophage Polarization and Function. J Leukoc Biol (2020) 108(3):983-98. doi: 10.1002/JLB.1A0420012RR

83. Zhu R, Huang YH, Tao Y, Wang SC, Sun C, Piao HL, et al. Hyaluronan UpRegulates Growth and Invasion of Trophoblasts in an Autocrine Manner via PI3K/AKT and MAPK/ERK1/2 Pathways in Early Human Pregnancy. Placenta (2013) 34(9):784-91. doi: 10.1016/j.placenta.2013.05.009

84. Wang S, Sun F, Han M, Liu Y, Zou Q, Wang F, et al. Trophoblast-Derived Hyaluronan Promotes the Regulatory Phenotype of Decidual Macrophages. Reproduction (2019) 157(2):189-98. doi: 10.1530/REP-18-0450

85. Tavianatou AG, Caon I, Franchi M, Piperigkou Z, Galesso D, Karamanos NK. Hyaluronan: Molecular Size-Dependent Signaling and Biological Functions in Inflammation and Cancer. FEBS J (2019) 286(15):2883-908. doi: $10.1111 /$ febs.14777

86. Rayahin JE, Buhrman JS, Zhang Y, Koh TJ, Gemeinhart RA. High and Low Molecular Weight Hyaluronic Acid Differentially Influence Macrophage Activation. ACS Biomater Sci Eng (2015) 1(7):481-93. doi: 10.1021/ acsbiomaterials.5b00181

87. Shi Q, Zhao L, Xu C, Zhang L, Zhao H. High Molecular Weight Hyaluronan Suppresses Macrophage M1 Polarization and Enhances IL-10 Production in 
PM2.5-Induced Lung Inflammation. Molecules (2019) 24(9):1766. doi: $10.3390 /$ molecules 24091766

88. Wakx A, Nedder M, Tomkiewicz-Raulet C, Dalmasso J, Chissey A, Boland S, et al. Expression, Localization, and Activity of the Aryl Hydrocarbon Receptor in the Human Placenta. Int J Mol Sci (2018) 19(12):3762. doi: 10.3390/ ijms19123762

89. Wu Y, Chen X, Chang X, Huang YJ, Bao S, He Q, et al. Potential Involvement of Placental AhR in Unexplained Recurrent Spontaneous Abortion. Reprod Toxicol (2016) 59:45-52. doi: 10.1016/j.reprotox.2015.11.005

90. Meng YH, Zhou WJ, Jin LP, Liu LB, Chang KK, Mei J, et al. RANKLMediated Harmonious Dialogue Between Fetus and Mother Guarantees Smooth Gestation by Inducing Decidual M2 Macrophage Polarization. Cell Death Dis (2017) 8(10):e3105. doi: 10.1038/cddis.2017.505

91. Bruno V, Lindau R, Jenmalm MC, Ticconi C, Piccione E, Pietropolli A, et al. First-Trimester Trophoblasts Obtained by Chorionic Villus Sampling Maintain Tolerogenic and Proteomic Features in Successful Pregnancies Despite a History of Unexplained Recurrent Pregnancy Loss. Am J Reprod Immunol (2020) 84(6):e13314. doi: 10.1111/aji.13314

92. Castro AS, Alves CM, Angeloni MB, Gomes AO, Barbosa BF, Franco PS, et al. Trophoblast Cells are Able to Regulate Monocyte Activity to Control Toxoplasma Gondii Infection. Placenta (2013) 34(3):240-7. doi: 10.1016/ j.placenta.2012.12.006

93. Tannetta D, Masliukaite I, Vatish M, Redman C, Sargent I. Update of Syncytiotrophoblast Derived Extracellular Vesicles in Normal Pregnancy and Preeclampsia. J Reprod Immunol (2017) 119:98-106. doi: 10.1016/ j.jri.2016.08.008

94. Statello L, Maugeri M, Garre E, Nawaz M, Wahlgren J, Papadimitriou A, et al. Identification of RNA-Binding Proteins in Exosomes Capable of Interacting With Different Types of RNA: RBP-Facilitated Transport of RNAs Into Exosomes. PloS One (2018) 13(4):e0195969. doi: 10.1371/ journal.pone. 0195969

95. Rezaie J, Rahbarghazi R, Pezeshki M, Mazhar M, Yekani F, Khaksar M, et al. Cardioprotective Role of Extracellular Vesicles: A Highlight on Exosome Beneficial Effects in Cardiovascular Diseases. J Cell Physiol (2019) 234 (12):21732-45. doi: 10.1002/jcp.28894

96. Salomon C, Torres MJ, Kobayashi M, Scholz-Romero K, Sobrevia L, Dobierzewska A, et al. A Gestational Profile of Placental Exosomes in Maternal Plasma and Their Effects on Endothelial Cell Migration. PloS One (2014) 9(6):e98667. doi: 10.1371/journal.pone.0098667

97. Sarker S, Scholz-Romero K, Perez A, Illanes SE, Mitchell MD, Rice GE, et al. Placenta-Derived Exosomes Continuously Increase in Maternal Circulation Over the First Trimester of Pregnancy. J Transl Med (2014) 12:204. doi: 10.1186/1479-5876-12-204

98. Mincheva-Nilsson L, Baranov V. Placenta-Derived Exosomes and Syncytiotrophoblast Microparticles and Their Role in Human
Reproduction: Immune Modulation for Pregnancy Success. Am J Reprod Immunol (2014) 72(5):440-57. doi: 10.1111/aji.12311

99. Orozco AF, Jorgez CJ, Ramos-Perez WD, Popek EJ, Yu X, Kozinetz CA, et al. Placental Release of Distinct DNA-Associated Micro-Particles Into Maternal Circulation: Reflective of Gestation Time and Preeclampsia. Placenta (2009) 30(10):891-7. doi: 10.1016/j.placenta.2009.06.012

100. Atay S, Gercel-Taylor C, Suttles J, Mor G, Taylor DD. Trophoblast-Derived Exosomes Mediate Monocyte Recruitment and Differentiation. Am J Reprod Immunol (2011) 65(1):65-77. doi: 10.1111/j.1600-0897.2010.00880.x

101. Atay S, Gercel-Taylor C, Taylor DD. Human Trophoblast-Derived Exosomal Fibronectin Induces Pro-Inflammatory IL-1beta Production by Macrophages. Am J Reprod Immunol (2011) 66(4):259-69. doi: 10.1111/ j.1600-0897.2011.00995.x

102. Holder B, Jones T, Sancho Shimizu V, Rice TF, Donaldson B, Bouqueau M, et al. Macrophage Exosomes Induce Placental Inflammatory Cytokines: A Novel Mode of Maternal-Placental Messaging. Traffic (2016) 17(2):168-78. doi: $10.1111 /$ tra. 12352

103. Ying X, Jin X, Zhu Y, Liang M, Chang X, Zheng L. Exosomes Released From Decidual Macrophages Deliver miR-153-3p, Which Inhibits Trophoblastic Biological Behavior in Unexplained Recurrent Spontaneous Abortion. Int Immunopharmacol (2020) 88:106981. doi: 10.1016/j.intimp. 2020.106981

104. Ding J, Zhang Y, Cai X, Zhang Y, Yan S, Wang J, et al. Extracellular Vesicles Derived From M1 Macrophages Deliver miR-146a-5p and miR-146b-5p to Suppress Trophoblast Migration and Invasion by Targeting TRAF6 in Recurrent Spontaneous Abortion. Theranostics (2021) 11(12):5813-30. doi: $10.7150 /$ thno. 58731

Conflict of Interest: The authors declare that the research was conducted in the absence of any commercial or financial relationships that could be construed as a potential conflict of interest.

Publisher's Note: All claims expressed in this article are solely those of the authors and do not necessarily represent those of their affiliated organizations, or those of the publisher, the editors and the reviewers. Any product that may be evaluated in this article, or claim that may be made by its manufacturer, is not guaranteed or endorsed by the publisher.

Copyright $\odot 2021$ Ding, Zhang, Cai, Diao, Yang and Yang. This is an open-access article distributed under the terms of the Creative Commons Attribution License (CC BY). The use, distribution or reproduction in other forums is permitted, provided the original author(s) and the copyright owner(s) are credited and that the original publication in this journal is cited, in accordance with accepted academic practice. No use, distribution or reproduction is permitted which does not comply with these terms. 